\title{
Pulsed differential calorimetry of Zn-doped LSCO cuprates
}

\author{
G. G. Basilia, G. A. Kharadze, K. A. Kvavadze, and M. M. Nadareishvili \\ Institute of Physics, Georgian Academy of Science, Tbilisi, Georgia \\ E-mail: gogi@iph.hepi.edu.ge \\ D. F. Brewer, G. Ekosipedidis, and A. L. Thomson \\ University of Sussex, Falmer, Brighton BN1 9QJ, U.K. \\ E-mail: d.f.brewer@sussex.ac.uk \\ Received February 11, 1998
}

\begin{abstract}
The low temperature heat capacity measurements on LSCO samples containing $\mathrm{Zn}$ impurities have been performed by means of pulsed differential calorimetry technique. The interpretation of the $\mathrm{Zn}$ concentration dependence of the residual $\gamma$-coefficient in the linear temperature part of $C_{\mathrm{el}}(T)$ at $T<<T_{c}$ is consistent with a model based on the assumption of the $d$-wave symmetry of the order parameter of these superconducting copper oxides.
\end{abstract}

PACS: 74.72.Dn, 74.25.Bt

\section{Introduction}

A great deal of effort has been applied to establish the symmetry properties of the ordered superconducting states of high- $T_{c}$ cuprates. In contrast to the superfluid phases of liquid ${ }^{3} \mathrm{He}$, for which the symmetries of the order parameters were established almost immediately after their discovery, the symmetry properties of the ordered states of high- $T_{c}$ superconductors are still under debate after ten years of considerable experimental and theoretical activity. What is, however, now firmly established is that the Cooper pairs in high- $T_{c}$ cuprates are in a spin-singlet state, and the main focus has become concentrated on the orbital structure of the order parameter [1].

A considerable amount of information accumulated during recent years has clearly shown that the magnitude of the gap function $|\Delta(\mathbf{k})|$ of the high- $T_{c}$ superconducting copper oxides is highly anisotropic in $\mathbf{k}$-space, but it is still necessary to explore the behavior of its phase which distinguishes, in particular, the $s$-wave-like and the $d$-wave-like properties of the Cooper-pair wave functions. Accordingly, phase-sensitive experiments on the order parameter have acquired special importance and among these the performance of Josephson-type measurements has proved to be the most direct way to establish the order parameter phase behavior. During the last few years these experiments have given valuable information about the orbital symmetry of $\Delta(\mathbf{k})$ in high- $T_{c}$ superconductors [2] with strong support for the proponents of the $d_{x^{2}-y^{2}}$-type behavior of the Cooper-pair wave-function, although some controversy is still present, possibly connected with distortion of the pure $d$-wave-like order parameter near the crystal boundaries [3].

Among other order-parameter phase-sensitive properties a response of the superconducting state to pair-breaking scattering events on various pointlike defects, localized on the conducting $\mathrm{CuO}_{2}$ planes of the high- $T_{c}$ superconducting cuprates, has also proved to be of great value. In contrast to the $s$-wave-like case the non-magnetic scattering centers strongly destroy the ordered state with $d_{x^{2}-y^{2}}$ symmetry [4-7]. This fact is intimately connected to the quantity $\langle\Delta(\mathbf{k})\rangle-$ i.e., the average value of the superconducting gap function across the Fermi surface. In the case of Cooper pairing in the $d_{x^{2}-y^{2}}$ channel, we have

$$
\Delta_{d}(\mathbf{k})=\Delta(T)\left(\cos a k_{x}-\cos a k_{y}\right)
$$

and $\langle\Delta(\mathbf{k})\rangle=0$. This property of the $d$-wave state opens the way to a strong suppression of $T_{c}$ by non-magnetic impurities (compare with Ref. 8) - 
an effect observed for various kinds of scattering centers introduced into high- $T_{c}$ cuprates, including those which are generated as a result of a radiation damage [9].

Another important effect of non-magnetic scattering centers on the properties of the $d$-wave paired state is the gradual filling of quasiparticle density of states near the Fermi level with increase in concentration of impurities. The gap function given by the Eq. (1) possesses nodes, $\Delta(\mathbf{k})=0$, along four lines on the cylindrical Fermi surface and due to this property the density of states $N_{f}$ at the Fermi level is zero in the pure limit with the infinite quasiparticle mean free path. In the presence of an impurity scattering (at a finite value of the meanfree path $l$ ) the Fermi-level density of states $N_{f} \neq 0$ and increases with a decrease of $l$, as has been predicted in a number of theoretical investigations [5-7]. These observations are in a sharp contrast with what is expected in the case of an $s$-wave-like Cooper pairing with $\langle\Delta(\mathbf{k})\rangle \neq 0$. This state may also have very pronounced anisotropy in the $\left(k_{x}, k_{y}\right)$ plane with a large difference between $\Delta_{\max }$ and $\Delta_{\min }$. One can even imagine an $s$-wave-like state with «accidental» zeros $\Delta_{\min }=0$ along the same nodal lines on the Fermi surface as in the $d_{x^{2}-y^{2}}$ pairing case. This rather artificial (but instructive) situation is described by a gap function with the form

$$
\Delta_{s}(\mathbf{k})=\Delta(T)\left|\cos a k_{x}-\cos a k_{y}\right|
$$

which again has $N_{f}=0$ in the pure limit. In spite of the similar magnitudes of the gap functions in Eqs. (1) and (2), the response to impurity scattering of a superconducting state with even symmetry, corresponding to Eq. (2), is radically different from what has just been described for the $d_{x^{2}-y^{2}}$ state, whose symmetry is odd. This happens because the impurity scattering events probe the phase properties of the order parameter and the phases of the gap functions given by Eqs. (1) and (2) behave quite differently. It can be shown $[6,7]$ that, for a superconductor with an $s$-wave-like pairing described by Eq. (2), instead of filling the quasiparticle states at the Fermi level, a finite gap $\varepsilon_{g}$ in the density of states grows up as a result of impurity scattering. In a more realistic $s$-wave-like case with $\Delta_{\min } \neq 0$ the impurity scattering pushes the gap to a value $\varepsilon_{g}>\Delta_{\text {min }}$ which simply means that in this case the impurities tend to smear out any initial in-plane anisotropy of the superconducting states. These very different responses of superconductors with conventional (s-wave-like) and unconventional ( $d$ wave-like) order parameters to the phase-sensitive impurity scattering events have proved to be a good probe of the symmetry of the order parameter in the high- $T_{c}$ cuprates.

A drastic difference in the quasiparticle spectral density near the Fermi energy for impurity-containing $s$-wave-like and $d$-wave-like ordered states has an immediate influence on the character of the low temperature behavior of the specific heat of superconductors providing one more possibility of exploring the symmetry properties of the high- $T_{c}$ copper oxide systems [10,11]. Although heat capacity measurements contain less direct information than Josephson experiments, they probe bulk properties of samples and are not sensitive to possible distortions of the order-parameter symmetry near the crystal boundaries.

\section{Experimental method}

We have performed low temperature heat capacity measurements on a family of LSCO samples doped with $\mathrm{Zn}$ by means of pulsed differential calorimetry technique. When using a differential method one measures the difference $\Delta C$ of the heat capacities of the sample of interest and the reference sample with known $C(T)$. Continuous and pulsed heating regimes can be used. The former method has been successfully applied in recent investigations of fundamental properties of a number of high- $T_{C}$ superconductors (see, for example, Refs. 12,13). The pulsed differential calorimeter designed by us [14] combines the high sensitivity of the continuous heating type differential calorimeter and the high accuracy of a classical pulsed calorimeter.

The measuring unit consists of two identical cells holding the sample of interest and the reference sample. The cells are connected by means of a thermal link so that before application of a heat pulse of duration $\Delta t$, as well as after some relaxation time $\tau>>\Delta t$, the two samples are in thermal equilibrium. At all stages adiabatic conditions are maintained.

The time evolution of the sample temperature is given by the equations

$$
\begin{aligned}
& T_{1}(t)-T_{i}=\frac{1}{C_{1}} \int_{0}^{t} \dot{Q}\left(t^{\prime}\right) d t^{\prime}, \\
& T_{2}(t)-T_{i}=\frac{1}{C_{2}} \int_{0}^{t} \dot{Q}\left(t^{\prime}\right) d t^{\prime},
\end{aligned}
$$


where $T_{1}(0)=T_{2}(0)=T_{i}$ is an initial temperature (before the heat pulse) and the heat-power input $\dot{Q}=I V$ is supplied simultaneously to both samples by means of an electric heater. From (3) it is seen that

$$
C_{1} \Delta T=I V \Delta t-\Delta Q, \quad C_{2} \Delta T=I V \Delta t+\Delta Q,
$$

where $\Delta T=T_{f}-T_{i}$ and $\Delta Q=K \int_{0}^{\infty} \delta T(t) d t$ is an amount of heat transferred between the samples through the thermal link (with thermal conductivity $K$ ) due to the temperature difference $\delta T(t)$ present between the two thermal equilibrium states. Finally, the measured heat capacity difference $\Delta C=2 \Delta Q / \Delta T$.

\section{Low-temperature calorimetry of $\mathrm{La}_{2-x} \mathrm{Sr}_{x} \mathrm{Cu}_{1-y} \mathrm{Zn}_{y} \mathrm{O}_{4}$}

We have investigated a zinc-doped LSCO series of samples by carrying out accurate measurements on their specific heats using our pulsed differential calorimetry techniques. Six samples of $\mathrm{La}_{2-x} \mathrm{Sr}_{x} \mathrm{Cu}_{1-y} \mathrm{Zn}_{y} \mathrm{O}_{4}$ were studied, all with an optimal level of hole doping $(x=0.16)$, and with $\mathrm{Zn}$ concentrations of $y=0.00 ; 0.01 ; 0.02 ; 0.033 ; 0.045$ and 0.06 . The sample containing 6 at.\% of $\mathrm{Zn}$ was used as the reference sample since it was certainly not a superconductor and, indeed, was in a metallic state. The samples were fabricated by a standard solid-state reaction in which the initial combination of the constituent chemical compounds was done via solid state mixing and then the samples were sintered at a temperature of $1030 \mathrm{~K}$ while being subjected to a high pressure. The resulting sample pill was then ground up and the sinter process was repeated again in the presence of a high pressure. Subsequent measurements of the susceptibility showed that the samples with $\mathrm{Zn}$ concentrations of $0.00,0.01$ and 0.02 had superconducting transition temperatures of $\sim 38 \mathrm{~K}, \sim 26 \mathrm{~K}$ and $\sim 15 \mathrm{~K}$, respectively. The samples with concentrations of 0.033 and 0.045 , on the other hand, displayed no superconducting transitions for temperatures down to $4.2 \mathrm{~K}$, which was the lowest temperature of our susceptibility measurements.

In the results reported below, the differential specific heat

$$
\Delta C(y, T)=C(y, T)-C(0.06, T)
$$

of the five samples with $y=0.00 ; 0.01 ; 0.02 ; 0.033$ and 0.045 were measured over the temperature in-

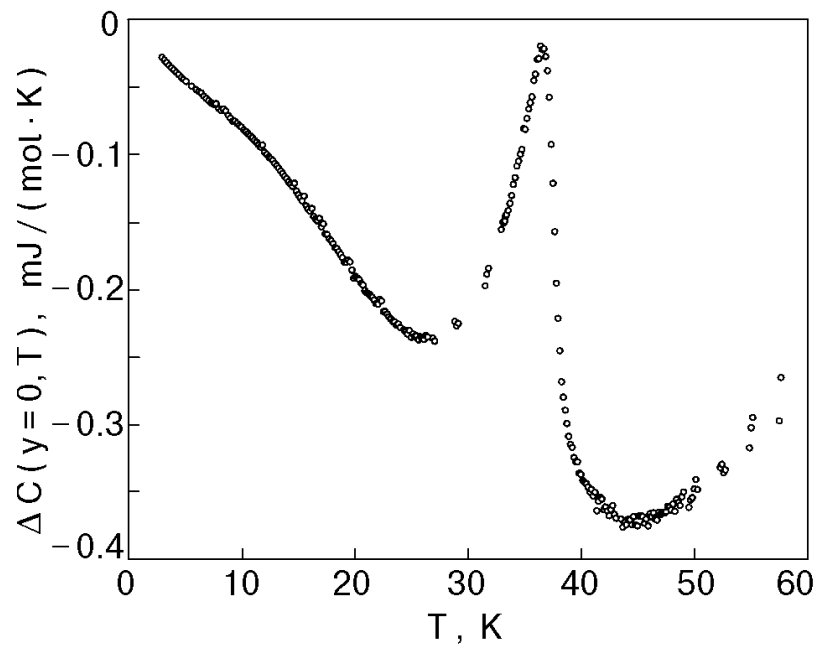

Fig. 1. Raw data for $\Delta C(y=0, T)$. A pronounced peak is clearly seen at $T_{c} \approx 37 \mathrm{~K}$.

terval 2-60 K, where the sample with the $0.06 \mathrm{Zn}$ concentration was being used as the reference sample. As an example, the raw data for the sample which contained no zinc, $\Delta C(0, T)$, are presented in Fig. 1 where a pronounced peak at the superconducting transition temperature, $T_{c} \cong 37 \mathrm{~K}$, for this nominally pure sample $(y=0)$ is evident.

The specific heat difference $\Delta C$ can be represented as a sum of electronic and phonon contributions:

$$
\Delta C=\Delta C_{\mathrm{el}}+\Delta C_{\mathrm{ph}} .
$$

Since $\mathrm{Zn}$ is isoelectronic with the $\mathrm{Cu}$ for which it is substituted, we may assume that the contribution $\Delta C_{\text {el }}$ will, in the normal state, depend only upon the hole concentration $x$ (and not upon $y$ ). This contribution is a term which is linear in temperature, $\gamma_{N} T$, where $\gamma_{N}$ is the Sommerfeld coefficient, and it is convenient to eliminate this contribution from the measured values of the quantity $\Delta C(y, T) / T$. For the reference sample, in its metallic state, the Sommerfeld coefficient $\gamma_{N}$, which could be obtained from the linear term in the specific heat, can be considered to remain constant over the whole temperature range and, therefore, for the sample of consentration $x=0.16$ we have put $\gamma_{N}=$ $=10 \mathrm{~mJ} /\left(\mathrm{mol} \cdot \mathrm{K}^{2}\right)$, in accordance with known results. Then, having settled upon this value for $\gamma_{N}$, we are able to obtain values for the function $\Delta \tilde{C} / T=\Delta C / T+\gamma_{N}$, which are presented in Fig. 2 for the samples with concentrations $y=0.00,0.01$ and 0.02 , and these clearly show the suppression produced in the transition temperature $T_{c}$ by the addition of $\mathrm{Zn}$ impurities. 


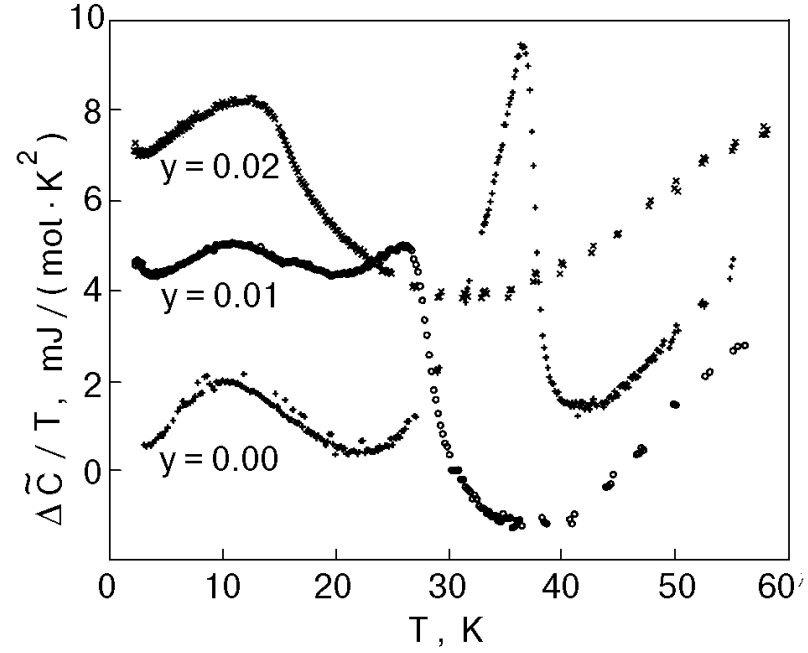

Fig. 2. Temperature dependence of $\Delta \tilde{C} / T=\Delta C / T+\gamma_{N}$ for three samples with $\mathrm{Zn}$ concentration $y=0.00 ; 0.01$ and 0.02 .

As was mentioned in the introduction, besides the suppression of $T_{c}$, the impurity scattering has a profound influence on the low temperature $\left(T<T_{c}\right)$ properties of a superconducting state with strong anisotropy of the gap function $\Delta(\mathbf{k})$ especially in the case of an unconventional (singular) behavior of its phase (as for $d$-wave Cooper pairing). In the low temperature domain $\Delta \tilde{C} / T$ can be represented as

$$
\frac{\Delta \tilde{C}}{T}=\gamma(y)+\Delta \beta T^{2}
$$

where the residual $\gamma(y)$ should be zero for $y=0$ in the case of the $d_{x^{2}-y^{2}}$ symmetric order parameter since $\gamma(y) \propto N_{f}(y)$ and $N_{f}(0)=0$ due to the nodes of $\Delta(\mathbf{k})$ on the Fermi surface. The $T^{2}$ term in (7), in addition to the phonon contribution, is expected to

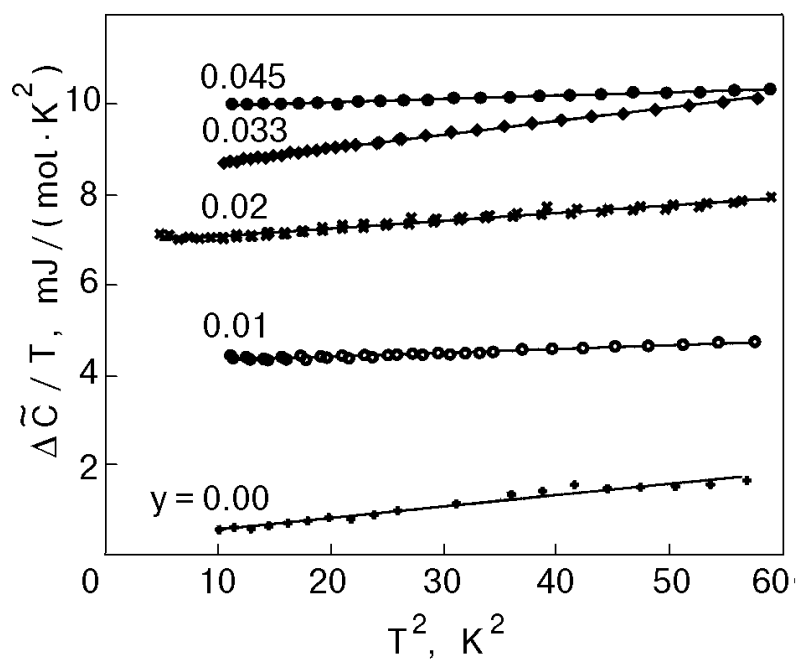

Fig. 3. Plots of $\Delta \tilde{C} / T$ as a function of $T^{2}$ in the low-temperature region $(T<8 \mathrm{~K})$.

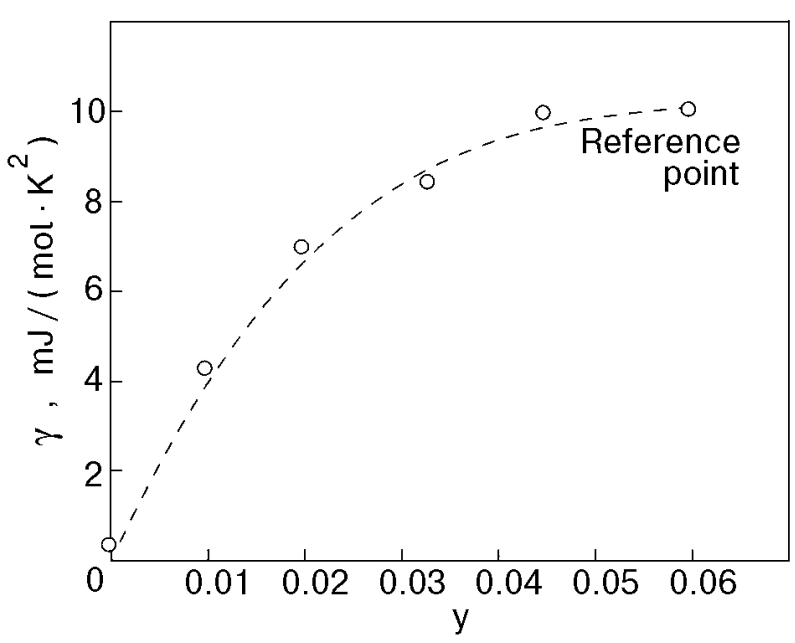

Fig. 4. Dependence of $\gamma$-coefficient on the $\mathrm{Zn}$ concentration.

have an electronic contribution in the case of a $d$-wave like order parameter [5].

Having plotted $\Delta \tilde{C} / T$ as a function of $T^{2}$, as shown in Fig. 3, we are able to find $\gamma(y)$ from the intercepts on this graph. The dependence of $\gamma$ at low temperatures on the $\mathrm{Zn}$ concentration is shown in Fig. 4. It is seen that $\gamma(y)$ increases rapidly with $y$ and then levels out for $y>0.04$. This behavior is consistent with the earlier measurements [10,11] which have been interpreted in terms of the $d_{x^{2}-y^{2-}}$ wave Cooper pairing model, predicting a gradual filling of the density of states at the Fermi level.

We have to keep in mind that, according to the $d$-wave pairing scenario, the temperature dependence given by Eq. (7) is expected for temperatures $T<T^{*}$, where $T^{*}$ is a disorder-dependent crossover temperature. In the temperature range $T^{*}<T<<T_{c}$ a term with a linear dependence on $T$ should appear in Eq. (7) due to a linear dependence of the quasiparticle density of states on the excitation energy $\varepsilon=\left|E-E_{F}\right|$ at low values for these energies $\left(\varepsilon^{*}<\varepsilon<<\Delta\right)$. Below $T^{*}$ the impurity induced deviation from this linear dependence takes over [15]. In the case of a sample with no impurities we should have $\gamma(0)=0=T^{*}$ and a contribution with a linear dependence on temperature should be present for temperatures $T<<T_{c}(\Delta \tilde{C} / T=\alpha T+$ $+\Delta \beta_{\mathrm{ph}} T^{2}$ ). We note that, according to our data, $\gamma(0) \neq 0$ (although it is rather small) indicating that in our nominally pure sample $(y=0)$ there is some amount of disorder and $T^{*} \neq 0$. This could be the cause of optimal hole doping where the $\alpha$-coefficient is expected to be the smallest and $T^{*}$ should be the largest at a given impurity scattering rate.

In conclusion, using pulsed differential calorimetry technique we have confirmed that the scattering of quasiparticles on $\mathrm{Zn}$ impurities present in 
LSCO high- $T_{c}$ superconductor has a pronounced influence on low-temperature thermodynamic properties. An interpretation of the $\mathrm{Zn}$-concentration dependence of the residual $\gamma$-coefficient of the linear temperature part of $C_{\mathrm{el}}(T)$ is consistent with a model based on the assumption of the $d$-wave symmetry of the order parameter of these superconducting copper oxides.

This work was partly supported by the INTAS Grant N1010-CT93-0046.

The authors are indebted to J.W. Loram for discussion of our experimental results and for providing us with the results of his own measurements prior to publication.

1. D. J. Scalapino, Phys. Rep. 250, 331 (1995).

2. D. J. Van Harlingen, Rev. Mod. Phys. 67, 515 (1995).

3. S. R. Bahcall, Phys. Rev. Lett. 76, 3634 (1996).

4. R. J. Radtke, K. Levin, H. B. Schuttler, and M. R. Norman, Phys. Rev. B48, 653 (1993).

5. T. Hotta, J. Phys. Soc. Jpn. 62, 274 (1993).
6. L. S. Borkovski and P. J. Hirschfeld, Phys. Rev. B49, 15404 (1994).

7. R. Fehrenbacher and M. R. Norman, Phys. Rev. B50, 3495 (1994).

8. A. I. Larkin, Pisma ZhETF 2, 205 (1995) [JETP Letters 2 , 130 (1965)].

9. E. M. Jackson, B. D. Weaver, G. P. Summers, P. Shapiro, and E. A. Burke, Phys. Rev. Lett. 74, 3033 (1995); S. K. Tolpygo, J.-Y. Lin, M. Gurvitch, S. Y. Hou, and J. M. Phillips, Phys. Rev. B53, 12454 (1996).

10. N. Momono and M. Ido, Physica C264, 311 (1996); N. Momono, M. Ido, T. Nakano, M. Oda, Y. Okajima, and K. Yamaya, Physica C233, 395 (1994).

11. M. Hiroi, H. Sato, M. Sera, and Kobayashi, Physica C235/240, 1779 (1994).

12. J. W. Loram, K. A. Mirza, J. R. Cooper, and W. Y. Liang, Phys. Rev. Lett. 71, 1740 (1993).

13. J. W. Loram, K. A. Mirza, J. R. Cooper, N. Athanassopoulou, and W. Y. Liang, 10th Anniversary HTS Workshop, Houston, March (1996).

14. K. A. Kvavadze and M. M. Nadareishvili, Patent N1610415 (1991).

15. P. J. Hirschfeld, W. O. Putikka, and D. J. Scalapino, Phys. Rev. Lett. 71, 3705 (1993); P. J. Hirschfeld and N. Goldenfeld, Phys. Rev. B48, 4219 (1993). 